\title{
Inherited disorder: pelger-huët anomaly
}

\begin{abstract}
Unfamiliarity with the appearance of immature neutrophils and mature neutrophils with bilobed nuclei and coarse clumping of the nuclear chromatin can lead to their erroneous classification. Presence of Pelger-Huët cells might be mistaken for a shift to the left, which represents an increase in the number of un-segmented (band) neutrophils in the circulation. Pelger-Huët anomaly, rather rare disorder is an inherited failure of the nuclei of neutrophils (and also eosinophils) to mature to the normal segmented form. PHA occurs secondary to mutations in the lamin B receptor. Consequently, a left shift always appears to be present. However, distinguishing PHA with acquired or pseudo-Pelger-Huët anomaly (PPHA), which has very similar morphologic characteristics, but it is associated with different pathological states, is very important.
\end{abstract}

Volume 4 Issue 4 - 2017

\author{
Nejra Hadžimusić \\ Department of Pathophysiology, University of Sarajevo, Bosnia \\ and Herzegovina
}

Correspondence: Nejra Hadžimusić, Assistant Professor, Department of Pathophysiology, Veterinary Faculty, University of Sarajevo, 7l 000 Sarajevo, Bosnia and Herzegovina,

Email nejra.hadzimusic@vfs.unsa.ba

Received: January 07, 2017 | Published: May II, 2017

Keywords: granulocytes, pelger-huët anomaly, pseudo-pelger-huët

Abbreviations: AML, acute myeloid leukemia; $\mathrm{CBC}$ complete blood count; CML, chronic myeloid leukemia; HIV, human immunodeficiency virus; IFN-g, interferon gamma; INM, inner nuclear membrane; LBR, lamin b receptor; MDS, myelodysplastic syndrome; NE, nuclear envelope; ONM, outer nuclear membrane; PHA, pelger-huët anomaly; PHA, pseudo-pelger-huët anomaly; TB, tuberculosis; TNF-a, tumor necrosis factor-alpha; WBC, white blood cell

\section{Introduction}

Morphologic evaluation of leukocytes by microscopic examination of a blood film is an essential part of the complete blood count (CBC). Nevertheless, finding a total leukocyte number within reference interval, presence of immature hyposegmented neutrophils in increased numbers (a left shift) suggests an inflammatory leukogram. However, neutrophils hyposegmentation also occurs with Pelger-Huët anomaly (PHA). ${ }^{1}$ The PHA has distinctive nuclear hyposegmentation of not only neutrophils, but also other granulocytes and monocytes, as well. The nuclear shape of affected granulocytes resembles bands, metamyelocytes or myelocytes however, the chromatin pattern appears aggregated and mature.

PHA is a benign inherited defect. $^{2}$ The neutrophil with characteristic appearance was first reported in two patients diagnosed with disseminated tuberculosis in 1928 by a Dutch hematologist-Karl Pelger. Observed neutrophils had bilobed nuclei, but also a coarse clumping of the nuclear chromatin. Pelger believed that the anomaly was associated with a poor prognosis in patients with tuberculosis because both patients died. ${ }^{3}$ Only three years later, Huët, who was also a Dutch, pronounced it as an inherited disorder. ${ }^{4}$ In fact, Huët was pediatrician and he recognized the "Pelger anomaly" in a 7-year-old girl with tuberculosis who recovered from her illness. ${ }^{3}$ Moreover, while examining smears of peripheral blood showed the Pelger anomaly in several of the child's relatives. Huët realize that the aberrant neutrophil morphology was a benign autosomal dominant inherited and surely not acquired change associated with a poor prognosis for tuberculosis. The Pelger-Huët anomaly is considered to be a familial anomaly and it is not connected with any pathologic state. In humans the PHA prevalence is between $0.01 \%$ and $0.1 \%$, the anomaly affects the white people, black and Asian in all ages and in equal ratio between males and females. ${ }^{5}$ The distribution of the anomaly is worldwide. The PHA is also present in other mammals such as mice, ${ }^{5}$ dogs,${ }^{6}$ cats, ${ }^{7}$ horses ${ }^{8}$ and rabbits. ${ }^{9}$ In humans, it is found rather rarely in a heterozygous state, according to Shetty et al..$^{10}$ in about one of every 5000 subjects. An incidence of one in 6000 is reported by Ayan et al. ${ }^{11}$ Presumed homozygous individuals have ovoid neutrophil nuclei, as well as varying degrees of developmental delay, epilepsy and skeletal abnormalities. ${ }^{12}$

The human neutrophil has a lobulated nucleus containing condensed chromatin. Its distinct nuclear shape facilitates rapid diapedesis through vessel walls and chemotaxis through interstitial spaces, respectively. The nucleus is bounded by a membrane system called the nuclear envelope (NE), which consists of outer nuclear membrane (ONM) and the inner nuclear membrane (INM). ${ }^{3}$ It has been reported that the lamin $\mathrm{B}$ receptor (LBR) mutations are the cause of PHA in humans. ${ }^{13}$ The lamin B receptor is an evolutionary conserved, multifunctional protein. ${ }^{14}$ LBR is an inner nuclear membrane protein that anchors the lamina and the heterochromatin to the inner nuclear membrane and has been shown to be significant for the normal morphologic maturation of granulocytes. However, nuclei of neutrophils in patients with PHA are not segmented due to a complete or partial lack of functional LBR. ${ }^{15}$ Granulocyte function in affected individuals appears to be normal. ${ }^{16}$ The morphologic difference between heterozygous and homozygous phenotypes in PHA was initially discovered in rabbits and subsequently documented in human case studies. ${ }^{17}$ In rabbit studies, the observed phenotypes when cross breeding heterozygotes did not conform to the expected number, and this led the researchers to hypothesize that many of the homozygotes were actually dying in utero; a finding they based on the observation of skeletal dysplasia and other morphologic abnormalities in the surviving homozygous rabbits. ${ }^{17}$ In human studies, there has been documentation of cognitive impairment, lethal hydrops, ectopic calcifications, cardiac defects, and skeletal deformities in homozygous carriers of mutations in the LBR gene. Also, it has been reported that the autosomal recessive, congenitally lethal disorder HEM/Greenberg skeletal dysplasia was caused by a nonsense mutation on the LBR gene. Moreover, hypolobulated PHA cells predominated on the peripheral smear of a mother whose fetus was determined to have HEM/Greenberg dysplasia. ${ }^{14}$ 
Pelger-Huët anomaly is diagnosed by the characteristic appearance of neutrophils with dumbbell-shaped to oval or round nuclei. The nuclear chromatin pattern has a coarse, clumped, mature pattern. Thy cytoplasm has a clear, mature and nontoxic appearance. Normal bands and metamyelocytes of the same shape have finer chromatin patterns and a bluer, more immature-appearing cytoplasm. Despite the almost all of the neutrophils appearing to be bands or younger based on nuclear shape, there is no inflammation and the disorder lasts the life of the individual. The characteristic white blood cell (WBC) appearance should be recognized and these neutrophils should be reported as Pelger-Huët cells and not non segmented cells to avoid misdiagnosis by clinicians reading the $\mathrm{CBC}$, who may falsely conclude very serious inflammation.

PHA must be distinguished from acquired or pseudo-Pelger-Huët anomaly (PPHA), which has although very similar morphologic characteristics, but has been related with pathologic conditions such as myelodysplastic syndrome, myeloproliferative disease (classically chronic myeloid leukemia, CML; acute myeloid leukemia) or myelofibrosis, as well as with certain infections (human immunodeficiency virus (HIV), TB and mycoplasma) and drugs. ${ }^{11}$ In these conditions, nuclear bilobation is generally asymmetric and is present in roughly $25 \%$ (clonal disorders) to $50 \%$ (drug-induced) of granulocytes. ${ }^{1}$ An acquired neutrophil dysplasia similar to PPHA also characterized by hyposegmentation of the neutrophil nucleus and excessive chromatin clumping has been described in hematological diseases and in some clinical situations, especially under the effect of certain drugs like tacrolimus, ganciclovir, co-trimoxazole, itraconazole fludarabine, rituximab, citalopram and lorazepam. Distinguishing this benign acquired or autosomal-dominant type from other acquired or pseudo-Pelger-Huët anomaly PPHA observed in individuals with myelodysplasia, myeloid leukemia and bi-lineage acute lymphocytic leukemia is necessary given the fact that recent case reports included the misinterpretation of PHA $\ P$ PHA as a possible myeloproliferative disorder, leading to unnecessary investigations which may include bone marrow procedures. Kuriyama et al. ${ }^{18}$ conducted a research considering PPHA in myelodysplastic syndrome (MDS). The research included 50patients with MDS and 86 control subjects. PPHA was determined in 38patients with MDS (approximately 76\%), 25patients with acute myeloid leukemia (AML) $(50 \%)$ and 19 of patients with chronic myelogenous leukemia (CML) (36\%). The extent of neutrophils with pseudo-PHA morphology was $3.8 \%$ of all the cells in the peripheral blood. ${ }^{10}$

\section{Conclusion}

Multiple theories exist behind the pathogenesis of pseudoPHA. It may be a result of a clonal myeloid malignancy, such as myelodysplastic syndrome, but also it might be a secondary nonclonal change related to a variety of underlying causes, including infections and medications. The clonal proliferation of dysplastic cells that have acquired mutations in the LBR gene is one of the theories. ${ }^{17} \mathrm{~A}$ second possibility considers PPHA as an apoptotic neutrophil and discards it as an abnormality in neutrophil maturation. Interestingly, ultrastructure of almost every cell of pseudo-PH cells looked like mature cell undergoing apoptosis. Electron-microscopy of these cells revealed all characteristics of apoptosis: nucleus of cells were compact, segregations of chromatin moved toward the periphery of the nucleus and condensation of the cytoplasm, organelles showing abnormal shape. ${ }^{17}$ This would be in respect with the excess of proapoptotic factors including TNF-a and IFN-g founded in microenvironment within the bone marrow of patients with infection or dysplastic processes. ${ }^{19}$ A third theory is a result of a research conducted on patients with MDS and AML. The results showed 14patients with pseudo-PH, 13 of those had $17 \mathrm{p}$ deletions. ${ }^{17}$ Further researches in LBR and leukemia may shed more progression in understanding its pathophysiology.

\section{Acknowledgements}

None.

\section{Conflict of interest}

The author declares no conflict of interest.

\section{References}

1. Roberts T, Linenberger M. A case of benign Pelger-Huët anomaly. Blood. 2015;126(5):693.

2. Shah SS, Parikh RS, Vaswani LP, et al. Familial pelger-huet anomaly. Indian J Hematol Blood Transfus. 2016;32(Suppl 1):347-350.

3. Colella R, Hollensead SC. Understanding and recognizing the pelgerHuët anomaly. Am J Clin Pathol. 2012;137(3):358-366.

4. Thajudeen AS, Prasaad PR. The degree of dysplasia in neutrophils with a special note on pelger- huët anomaly. APALM. 2017;4(1):18-21.

5. Dangelo G. The Pelger-Huët Anomaly. From morphology to clinical suspicion. International Journal of Clinical Medicine Research. 2014;1(4):125-127.

6. Vale AM, Tomaz LR, Sousa RS, et al. Pelger-Huët anomaly in two related mixed-breed dogs. J Vet Diagn Invest. 2011;23(4):863-865.

7. Deshuillers P, Raskin R, Messick J. Pelger-Huët anomaly in a cat. Vet Clin Pathol. 2014;43(3):337-341.

8. Gill AF, Gaunt S, Sirninger J. Congenital Pelger-Huët anomaly in a horse. Vet Clin Pathol. 2006;35(4):460-462.

9. Supuka P, Mazensky D, Supukova A, et al. Homozygous Pelger-Huet anomaly in three different crossbred rabbits: a case report. Veterinární medicína. 2014;59(2):95-101.

10. Shetty VT, Mundle SD, Raza A. Pseudo Pelger-Huet anomaly in myelodysplastic syndrome: Hyposegmented apoptotic neutrophil? Blood. 2001;98(4):1273-1275.

11. Ayan MS, Abdelrahman AA, Khanal N, et al. Case of acquired or pseudoPelger-Huët anomaly. Oxf Med Case Reports. 2015;2015(4):248-250.

12. Hoffmann K, Dreger CK, Olins AL, et al. Mutations in the gene encoding the lamin B receptor produce an altered nuclear morphology in granulocytes (Pelger-Huët anomaly). Nat Genet. 2002;31(4):410-414.

13. Turner EM, Schlieker C. Pelger-Huët anomaly and Greenberg skeletal dysplasia: LBR-associated diseases of cholesterol metabolism. Rare Dis. 2016;4(1):e1241363.

14. Tsai PL, Zhao C, Turner E, et al. The Lamin B receptor is essential for cholesterol synthesis and perturbed by disease-causing mutations. Elife. 2016;5:e16011.

15. Rowat AC, Jaalouk DE, Zwerger M, et al. Nuclear envelope composition determines the ability of neutrophil-type cells to passage through micronscale constrictions. J Biol Chem. 2013;288(12):8610-8618.

16. Lukaszewska J, Allison RW, Stepkowska J. Congenital pelger-huët anomaly in a Danish/Swedish Farmdog: case report. Acta Vet Scand. 2011;53:14.

17. Cunningham JM, Patnaik MM, Hammerschmidt DE, et al. Historical perspective and clinical implications of the Pelger-Huët cell. Am J Hematol. 2009;84(2):116-119. 
18. Kuriyama K, Tomonaga M, Matsuo T, et al. Diagnostic significance of detecting pseudo-Pelger-Huët anomalies and micro-megakaryocytes in myelodysplastic syndrome. Br J Haematol. 1986;63(4):665-669.
19. Economopoulou C, Pappa V, Kontsioti F, et al. Analysis of apoptosis regulatory genes expression in the bone marrow $(\mathrm{BM})$ of adult de novo myelodysplastic syndromes (MDS). Leuk Res. 2008;32(1):61-69. 